\title{
Dynamics of anisotropic oxygen-ion migration in
}

\section{strained cobaltites}

Qinghua Zhang,,$\S \dagger$ Fanqi Meng,,+ Ang Gao,, Xinyan Li,, Qiao Jin,, , Shan Lin, , Shengru Chen, ${ }^{\ddagger, l}$ Tongtong Shang, ${ }^{\ddagger}$, Xing Zhang, ${ }^{\#}$ Haizhong Guo, $\nabla$ Can Wang, $\$, \circ$ Kui-juan

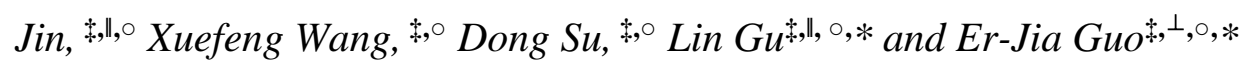

¥ Beijing National Laboratory for Condensed Matter Physics, Institute of Physics, Chinese Academy of Sciences, Beijing 100190, China

${ }^{\S}$ Yangtze River Delta Physics Research Center Co. Ltd., Liyang 213300, China

" School of Physical Sciences, University of Chinese Academy of Sciences, Beijing 100049,

China

${ }^{\perp}$ Center of Materials Science and Optoelectronics Engineering, University of Chinese Academy of Sciences, Beijing 100049, China

\# Beijing National Laboratory for Molecular Sciences, Key Laboratory of Molecular Nanostructure and Nanotechnology, Institute of Chemistry, Chinese Academy of Sciences, Beijing 100190, China.

$\nabla$ School of Physics and Microelectronics, Zhengzhou University, Zhengzhou 450001, China

${ }^{\circ}$ Songshan Lake Materials Laboratory, Dongguan, Guangdong 523808, China

E-mail: ejguo@iphy.ac.cn; 1.gu@iphy.ac.cn

$\dagger$ These authors contribute equally to this work.

KETWAORDS: oxygen vacancy channel; epitaxial strain; domain switching; oxygen-ion migration; in-situ STEM. 


\begin{abstract}
Orientation control of oxygen vacancy channel (OVC) is a highly desirable for tailoring oxygen diffusion as it serves fast transport channel in ion conductors, which is widespread exploited in solid-state fuel cells, catalysts, and ion-batteries. Direct observation of oxygen-ions hopping towards preferential vacant sites is a key to clarifying migration pathways. Here we report the anisotropic oxygen-ion migration mediated by strain in ultrathin cobaltites via in-situ thermal activation in an atomic-resolved transmission electron microscopy. Oxygen migration pathways are constructed on the basis of the atomic structure during the OVC switching, which is manifested as the vertical-to-horizontal OVC switching under tensile strain, but the horizontal-to-diagonal switching under compression. We evaluate the topotactic structural changes to OVC, determine the crucial role of tolerance factor for OVC stability and establish the strain-dependent phase diagram. Our work provides a practical guide for engineering OVC orientation that is applicable ionic-oxide electronics.
\end{abstract}




\section{TEXT}

Oxygen-ion migration in transition metal oxides has attracted an increasing interest in fundamental research and applications in solid oxide fuel cells, ${ }^{1-3}$ fast catalytic reaction, ${ }^{4}$ resistive switching memories, ${ }^{5}$ electrochemical sensing, ${ }^{6}$ and etc. The oxygen-ion migration in solids is ascribed to the hopping towards lower energetic vacant oxygen sites. Ordering of the oxygen vacancy $\left(V_{\ddot{o}}\right)$ emerges when their concentration reaches a fractional threshold in oxide parents, ${ }^{7}$ leads to the formation of the oxygen vacancy channel (OVC) $)^{8-11}$ and stimulates various emergent quantum phenomena. ${ }^{12-18}$ More importantly, the creation or reorientation of OVC introduce topotactic phase transition such as the transformation from perovskite to brownmillerite $(\mathrm{BM})$, resulting in dramatic change in physical and chemical properties including the metal-insulator transition, magnetic phase transition, and anisotropic ion

conduction. ${ }^{11,19-24}$ The OVC usually serves as a fast channel for oxygen vacancy diffusion, ${ }^{17,18}$ play the key role in regulating migration pathway of oxygen ions and dominating the dynamic response to external stimulus. Thus, controllable orientation of OVC is desirable for tailoring oxygen diffusion as its widespread exploited in solid-state fuel cells, catalysts, and ion-batteries. A thoughtful understanding of topochemical aspects of the oxygen-ion migration will give us further insight into the control parameters of oxygen-ion migration pathways and the utilization of OVC in designing solid oxygen-ion conductors. ${ }^{25-27}$

Strain engineering is a universal strategy to tune the atomic configuration of OVCs by directly altering the cation-oxygen bond length and oxygen vacancy formation energy $\left(E_{V o ̈}\right)$. Moving an oxygen ion out of its equilibrium position along a specific migration pathway to the neighboring vacant lattice site is determined by the strain state of epitaxial thin films. It is reported that the orientation of OVCs in cobaltites, ${ }^{28}$ chromates, ${ }^{29}$ and ferrites ${ }^{30}$ thin films can be readily controlled by strain. For instance, the periodicity and orientation of $V_{\ddot{o}}$ layers are parallel (perpendicular) to the interface when the films under compressive (tensile) strain. ${ }^{31}$ 
Recently, an unexpected zigzag-like $V_{\ddot{o}}$ ordering in a compressively strained $\mathrm{LaCoO}_{2.5}$ film has been found, whereas the unique structure cannot be formed through annealing a tensile-strained $\mathrm{LaCoO}_{3}\left(\mathrm{LCO}_{3}\right)$ film. ${ }^{32}$ Previously, the OVC formation under tensile strain has been studied by high-dose electron beam irradiation. ${ }^{33,34}$ Dynamic process in differently strained $\mathrm{LaCoO}_{\mathrm{x}}$ $\left(\mathrm{LCO}_{\mathrm{x}}\right)$ films towards further oxygen reduction to the metastable phases has not been visualized directly so far.

Here we report an operando transmission electron microscopy (TEM) investigation of dynamic structural evolution in $\mathrm{LCO}_{\mathrm{x}}$ films during the thermally annealing. We observe that the final-stabilized $\mathrm{LCO}_{2.5}$ phases are distinct when the pristine $\mathrm{LCO}_{3}$ films under compressive and tensile strain states. The OVC evolutions of strained $\mathrm{LCO}_{\mathrm{x}}$ films are recorded as a function of both annealing temperature and annealing time. By imaging the atomic-scale boundaries of OVC switching, this work provides the direct evidence of strain-mediated $V_{\ddot{o}}$ migration pathway from an octahedron. The associated lattice distortion and its strain dependency are quantified theoretically by mapping out the OVC phase diagram at the atomic scale.

\section{Strain dependence of OVCs' orientation in ultrathin cobaltites}

Previous theoretical and experimental work ${ }^{35-40}$ demonstrate that $E_{V o ̈}$ in perovskite-type oxides depends on the strain and is highly anisotropic. Tensile strain dramatically reduces $E_{V o ̈}$, whereas the compressive strain only changes $E_{V o ̈}$ slightly. We choose $\mathrm{LCO}_{3}$ as our protocol system due to the following two reasons. First of all, cobaltites have relatively low $E_{V \ddot{o}}(\sim 1.5$ $\mathrm{eV})$ and oxygen migration barrier $(\sim 0.8 \mathrm{eV})$, compared to other $3 d$ transition metal oxides. ${ }^{40}$ Secondly, earlier work shows that the stoichiometric $\mathrm{LCO}_{3}$ epitaxial films exhibit an unconventional strain relaxation behavior, resulting in the stripe-like domain patterns due to the

formation of OVCs. ${ }^{41-44}$ The pseudocubic (pc) lattice constant of bulk $\mathrm{LCO}_{3}$ is $a_{p c}=3.81 \AA$ (Figure 1a). Single-crystalline $\mathrm{SrTiO}_{3}(a=3.905 \AA)$ and $\mathrm{LaAlO}_{3}\left(a_{p c}=3.79 \AA\right)$ substrates may introduce $-0.5 \%$ (compressive) and $+2.5 \%$ (tensile) strain to the as-grown $\mathrm{LCO}_{3}$ films, respectively. Therefore, the $\mathrm{LCO}_{3}$ thin film is expected to exhibit the unique structural evolution 
of OVC under different strain states. ${ }^{45}$ We choose the $\mathrm{LaAlO}_{3}$ as capping layer on $\mathrm{LCO}_{3}$ films because it has a lower oxygen migration barrier $(\sim 0.63 \mathrm{eV})$ and higher oxygen vacancy formation energy (4.2-6.5 eV) than those of cobaltites. ${ }^{46-48}$ Therefore, the oxygen could extract out of the films without deteriorating the crystal structure of the capping layer. X-ray diffraction and reflectivity measurements confirm the highly epitaxial, coherently growth, and smooth interface/surface in the as-grown $\mathrm{LaAlO}_{3} / \mathrm{LCO}_{3}$ bilayers (Figure S1). The absence of periodic dark-strips in the pristine $\mathrm{LCO}_{3}$ films of both strain states indicate that all $\mathrm{LCO}_{3}$ films are nearly stoichiometric (Figure S2). The oxygen had been gradually extracted from $\mathrm{LCO}_{3}$ films by gradually increasing temperature at rate of $5{ }^{\circ} \mathrm{C} / \mathrm{min}$ in the ultrahigh vacuum environment ( $10^{-9}$ Torr) of STEM (Figure S3 and S4). Figures $1 \mathrm{~b}$ and 1c show the immediate high-angle annular dark-field (HAADF) STEM images of $\mathrm{LCO}_{2.67}$ phases under tensile and compressive strain states at an annealing temperature of $T_{\mathrm{a}}=400{ }^{\circ} \mathrm{C}$, respectively. As oxygen-ion escaped out of the $\mathrm{LCO}_{3}$ films, the increased "dark-stripes" can be visualized in both samples. The $\mathrm{LCO}_{2.67}$ phases contain alternatively stacked one $\mathrm{CoO}_{4}$ tetrahedral and two $\mathrm{CoO}_{6}$ octahedral layers. ${ }^{41}$ As shown in Figure $1 \mathrm{~b}$, tensile strain would favor the creation of ordered $V_{\ddot{o}}$ in the films along the out-of-plane direction because the $V_{\ddot{o}}$-related chemical expansion can reduce the lattice misfit strain. ${ }^{41-44}$ On the contrary, compressive strain leads to in-plane $V_{\ddot{o}}$ orderings, which is parallel to the interface due to the concomitant lattice elongation along the out-ofplane direction (Figure 1c).

Spontaneously, the ordered OVCs are formed within the tetrahedral $\mathrm{CoO}_{4}$ layer in every one-third atomic plane, ${ }^{49}$ producing the dark-stripe contrast in the HAADF images due to the increased La-La distance. Under tensile strain (on a $\mathrm{SrTiO}_{3}$ substrate), $V_{\ddot{o}}$ sites prefer to the ordered arrangement along the out-of-plane direction, resulting in vertical $V_{\ddot{o}}$ stripes mostly with $3 a$ periodicity (denoted as $3 a-\mathrm{LCO}_{2.67}$ hereafter). In contrast, horizontal $V_{\ddot{o}}$ stripes with $3 c$ periodicity (denoted as $3 c$ - $\mathrm{LCO}_{2.67}$ hereafter) appeared in the films grown on the $\mathrm{LaAlO}_{3}$ substrates, corresponding to the $V_{\ddot{o}}$ sites aligned in the in-plane $\mathrm{CoO}_{4}$ layers. 


\section{Evolution of OVCs in strained $\mathrm{LCO}_{\mathrm{x}}$ films}

The orientation of OVCs switches from the out-of-plane to the in-plane orientation when a small amount of oxygen vacancies was extracted under tensile strain at a slightly higher temperature of $450{ }^{\circ} \mathrm{C}$. As shown in Figure 2a, the horizontal $V_{\ddot{o}}$ stripe domains $\left(3 c-\mathrm{LCO}_{2.67}\right)$ firstly appear randomly in the $3 a-\mathrm{LCO}_{2.67}$ films and then propagate laterally. As increasing annealing time, the horizontal $V_{\ddot{o}}$ stripe domains emerge on the right side, and then moved towards the left region with vertical $V_{\ddot{o}}$ stripes within the tens of seconds (the minimum imaging time frame in scanning mode). It is hampered by an edge dislocation labeled by a blue triangle (Figure 2b). After annealing at $T_{\mathrm{a}}=450{ }^{\circ} \mathrm{C}$ around 2 minutes, the horizontal $V_{\ddot{o}}$ stripes crossed the edge dislocation and overspread the whole film. After $\sim 4$ minutes of high-temperature annealing, the $3 c-\mathrm{LCO}_{2.67}$ film gradually transforms into the brownmillerite (BM) phase with $2 c$ periodicity (denoted as $2 c$ - $\mathrm{LCO}_{2.5}$ ) and finally stabilizes at $2 c$ - $\mathrm{LCO}_{2.5}$ phase after 6 -minutesannealling time (Figures 2c and 2d). Please note that the OVCs' alignment switches when the $3 a-\mathrm{LCO}_{2.67}$ changes to $3 c-\mathrm{LCO}_{2.67}$ without changing oxygen content. We could extract the moving speed of domain boundary is $\sim 15 \mathrm{~nm} / \mathrm{mins}$. However, the domain boundaries change from vertical to horizontal alignment with further extracting oxygen to $2 c-\mathrm{LCO}_{2.5}$.

The evolution behavior of OVCs in $\mathrm{LCO}_{\mathrm{x}}$ films under compressive strain is dramatically different to that of a film under tensile strain. At $T_{\mathrm{a}}=500{ }^{\circ} \mathrm{C}$, we observe a new $V_{\ddot{o}}$ ordered phase with diagonal-aligned dark-stripes, which can be called n- $\mathrm{LCO}_{2.5}$ as reported in our recent work. ${ }^{32}$ The diagonal $V_{\ddot{o}}$ stripes firstly appear randomly in the $3 c-\mathrm{LCO}_{2.67}$ films as indicated by the inclined purple arrows in Figure 2e. As increasing annealing time, the n- $\mathrm{LCO}_{2.5}$ domains propagate laterally toward two sides in the moving speed of domain wall of $\sim 7 \mathrm{~nm} / \mathrm{mins}$, accompanied by the disappearance of horizontal $V_{\ddot{o}}$ stripes (Figures $2 \mathrm{f}$ and $2 \mathrm{~g}$ ). After around 6 minutes, the $3 c-\mathrm{LCO}_{2.67}$ completes the structural transformation to the $n-\mathrm{LCO}_{2.5}$ phase in the entire film (Figure 2h). Both HAADF and annular bright field (ABF) images of n-LCO 2.5 were recorded for identifying the atomic positions of each element (Figure S5). From ABF images, 
we could visualize the tilt and distortion of $\mathrm{CoO}_{5}$ square pyramids, which are dramatically distorted close to $V_{\ddot{o}}$ cites. The periodic OVCs along the diagonal direction were visualized directly, as indicated by bright contrast in ABF images. The dynamic process of dark stripe formation and evolution under thermal annealing exhibits completely different pathways depending on epitaxial strain.

\section{Atomic view of domain boundaries and strain distributions in $\mathrm{LCO}_{\mathrm{x}}$ films}

To reveal the atomic-resolved structural transition of OVCs, we compared the changes on the periodicity and orientation of $V_{\ddot{o}}$ stripes in the high-magnified HAADF images of $\mathrm{LCO}_{\mathrm{x}}$ films grown on $\mathrm{SrTiO}_{3}$ and $\mathrm{LaAlO}_{3}$ substrates, shown in Figures 3a and 3d, respectively. Figure $3 \mathrm{c}$ shows the domain boundary between $3 a-\mathrm{LCO}_{2.67}$ and $3 c-\mathrm{LCO}_{2.67}$. The atomic arrangement and structure model of domain boundary are shown in Figure $4 \mathrm{c}$. We find that the vertical $V_{\ddot{o}}$ stripes are not connected directly to the horizontal ones but always blocked by an octahedral layer, marked in red shadow. The possible migration path of oxygen ions is illustrated in Figure S6. The oxygen ions will migrate from diagonal octahedron to the nearby tetrahedron, resulting in the rearrangement of dark stripes from vertically-aligned to horizontally-aligned. Meanwhile, the oxygen ions flow from an octahedron to a tetrahedron within the $3 a-\mathrm{LCO}_{2.67}$ domains in order to keep an octahedral layer at the domain boundary. Macroscopically, the $3 c$ - $\mathrm{LCO}_{2.67}$ domains grow and $3 a-\mathrm{LCO}_{2.67}$ domains vanish. Finally, the dark stripes change its periodicity from $3 c$ to $2 c$ by increasing numbers of $V_{\ddot{o}}$. Eventually, the $3 c-\mathrm{LCO}_{2.67}$ undergoes a topotactic structural transformation into BM-phase $2 c-\mathrm{LCO}_{2.5}$. Strain evolution at the domain boundary between $3 a-\mathrm{LCO}_{2.67}$ and $3 c-\mathrm{LCO}_{2.67}$ was also quantitatively analyzed by La-La distance mapping (Figure 3b and Figure S7). An obvious strain distribution can be visualized by the color contrast of lattice constants. A uniform contrast around domain boundary suggests that the lattice mismatches have been well accommodated by horizontal stripes through the orientation switching of OVCs, indicating a smooth transition from $3 a-\mathrm{LCO}_{2.67}$ to $3 c$ - $\mathrm{LCO}_{2.67}$ domains. 
Figure $3 \mathrm{f}$ shows the high-magnified HAADF image around the domain boundary between $3 c-\mathrm{LCO}_{2.67}$ and n- $\mathrm{LCO}_{2.5}$. The horizontal $V_{\ddot{o}}$ stripes with $2 c$ and $3 c$ periodicities coexist in $\mathrm{LCO}_{2.67}$, indicating the oxygen content of $\mathrm{LCO}_{\mathrm{x}}$ films derivates from a stochiometric $\mathrm{LCO}_{2.67}$ and approaches towards $\mathrm{LCO}_{2.5}$. The horizontal $V_{\ddot{o}}$ stripes in $3 c-\mathrm{LCO}_{2.67}$ with alternative $\left[\mathrm{CoO}_{6}\right]$ octahedron and $\left[\mathrm{CoO}_{4}\right]$ tetrahedron layers switch to the uniformed $\left[\mathrm{CoO}_{5}\right]$ square pyramids in $\mathrm{n}-\mathrm{LCO}_{2.5}$. There is no blocking layer between two distinct domains. The horizontal $V_{\ddot{o}}$ stripes are connected directly to the diagonal $V_{\ddot{o}}$ site of $\mathrm{n}-\mathrm{LCO}_{2.5}$ phase, indicating a comparable short oxygen-ion migration pathway. In this case, the oxygen ions migrate from the neighbored octahedron and tetrahedron vertically (Figure S8), resulting in the formation of pyramids. Different to the tensile strained films, the n- $\mathrm{LCO}_{2.5}$ phase remain coherently strained by $\mathrm{LaAlO}_{3}$ substrate (Figure 3e and Figure S9). Although the in-plane and out-of-plane lattice constants changes alternatively along the diagonal direction within the n- $\mathrm{LCO}_{2.5}$ layer, the substrate's misfit strain can be perfectly transferred to the capping layers.

\section{Strain dependent oxygen migration and phase diagram of OVCs in cobaltites.}

To verify the influence of elastic strain on $E_{V o ̈}$ in $\mathrm{LaCoO}_{3}$ system, we performed the firstprinciples calculations based on density function theory (Figures $4 \mathrm{a}$ and $4 \mathrm{~b}$ ). $E_{V o ̈}$ reduces monotonously as the in-plane stain changes from compressive to tensile strain state. Experimentally, we indeed observe a lower transition temperature of $3 a-\mathrm{LCO}_{2.67}$ than that of $3 c-\mathrm{LCO}_{2.67}$. More importantly, the strain-dependent $E_{V \ddot{o}}$ determines the OVC orientation when the $V_{\ddot{o}}$ stripes form at the early stage. The phase transition from $3 a-\mathrm{LCO}_{2.67}$ to $3 c$ - $\mathrm{LCO}_{2.67}$ undergoes a OVC switching by 90 degrees. The oxygen ions rearrange within the films without changing oxygen content. Strain-dependent $\mathrm{OVC}$ in $3 a-\mathrm{LCO}_{2.67}$ and $3 c-\mathrm{LCO}_{2.67}$ is reasonable in terms of minimization of the elastic energy at the interface between films and substrates. We believe that the oxygen extracts out of the strained $\mathrm{LCO}_{3}$ films may have different migration pathways as the OVCs serve as oxygen ion passes. To form $3 a-\mathrm{LCO}_{2.67}$, the oxygen comes out of the capping layer directly in a short way and relative short time, whereas the oxygen migrates 
a long way horizontally to the edges of films to get out of the films resulting in the $3 c-\mathrm{LCO}_{2.67}$. This process takes a relative long time. We believe that the anisotropic oxygen-ion migration under strain would exhibit the different ionic conducting speed and efficiency.

Next, we will discuss how the oxygen-ion migration proceeds in $\mathrm{LCO}_{2.67}$ during the orientation switching of OVC. Two possible reaction processes at the atomic-scale can be deduced based on the atomic structures of domain boundaries (Figures $4 \mathrm{c}$ and $4 \mathrm{~d}$ ). When a $3 a-$ $\mathrm{LCO}_{2.67}$ is tensile-strained, the $\mathrm{O} 1$ shared by the $\mathrm{CoO}_{6}$ octahedron and $\mathrm{CoO}_{4}$ tetrahedron preferentially moves to a vacant site $\mathrm{Vo} 1$ in the neighbored $\mathrm{CoO}_{4}$ tetrahedron along the polyhedral edge with a migration barrier of $\sim 0.95 \mathrm{eV}$, then followed by multiple steps of ion migration along the O1-Vo1-Vo2 and O2-O1-Vo1 pathways. Finally, the oxygen ions migrate to a stable location and complete exchanges between the $\mathrm{CoO}_{6}$ octahedron and $\mathrm{CoO}_{4}$ tetrahedron as indicated by the blue double arrows in Figure 4c. Consequently, the orientation switching of OVC can be elucidated by cooperatively multiple octahedra-tetrahedra exchanges, as indicated by the schematics in Figure S6. In comparison, when a compressive strain is applied to $3 c-\mathrm{LCO}_{2.67}$, the migration barrier $(\sim 1.03 \mathrm{eV})$ is slightly larger than that of former case. This fact well-explains that a higher $V_{\ddot{o}}$ extraction temperature is needed for compressively strained $\mathrm{LCO}_{\mathrm{x}}$ films. The final $V_{\ddot{o}}$ pattern in $\mathrm{n}-\mathrm{LCO}_{2.5}$ films is in sharp contrast to that of $\mathrm{BM}-\mathrm{LCO}_{2.5}$ on $\mathrm{SrTiO}_{3}$ substrate. Interestingly, the oxygen-ion migration can be interpreted in an easier way based on the uniform $\mathrm{CoO}_{5}$ configuration, as shown in Figure 4d. The migration can be accompanied just by a successive O1-Vo1 and O2-O1 hopping, leading to a simultaneous transition from tetrahedron/octahedron into the $\mathrm{CoO}_{5}$ square pyramids. The neighboring octahedron would transform into $\mathrm{CoO}_{5}$ square pyramids by losing one $\mathrm{O} 3$ ion, as indicated by the green arrow. As a result, the OVC switches towards diagonal $V_{\ddot{o}}$ stripes can be elucidated by conserved octahedra-tetrahedra evolution and partial oxygen-ion loss, as summarized by the schematics in Figure S8. 
Based on the distinct OVC states and the orientation switching behaviors observed in $\mathrm{LCO}_{x}$ films grown on $\mathrm{SrTiO}_{3}$ and $\mathrm{LaAlO}_{3}$ substates, we draw a phase diagram of OVC in strained $\mathrm{LCO}_{3-\mathrm{x}}$ films is established as a function of misfit strain and oxygen content in Figure 4e. Epitaxial strain determines the initial orientation of $V_{\ddot{o}}$ stripes, as evidenced by the vertical stripes on the tensile strain states and the horizontal stripes on the compressive strain states. When the $\mathrm{LCO}_{\mathrm{x}}$ films are tensile-strained, the lattice structure firstly transits from $\mathrm{LCO}_{3}$ into the $3 a-\mathrm{LCO}_{2.67}$, then further transforms into the $3 c-\mathrm{LCO}_{2.67}$ state. Apparently, the OVC orientation concomitant switches during the topotactic structural transition. The OVC orientation maintains when the LCOx films transit into the $2 c-\mathrm{LCO}_{2.5}$. We believe that the OVC orientation switching in the tensile-strained $\mathrm{LCO}_{\mathrm{x}}$ films is attributed to the strain accommodation between the final state, i.e., $2 c-\mathrm{LCO}_{2.5}$, and STO substrates., yielding to a $+2.5 \%$ tensile strain. Of most interest, on the compressive strain regime, a new $\mathrm{LCO}_{2.5}$ phase emerged when a large number of oxygen-ions is extracted from the $3 c-\mathrm{LCO}_{2.67}$ phase, forming a completely new $V_{\ddot{o}}$ ordering configuration. The quantitative analysis on the $V_{\ddot{o}}$-induced chemical expansion is favorable for uncovering the mechanism of OVC orientation switching. As increasing the density of $V_{\ddot{o}}$ stripes, the large La-La distance $d_{V o}(4.39 \pm 0.05 \AA)$ in the $V_{\ddot{o}^{-}}$ ordered $\mathrm{CoO}_{4}$ tetrahedral layers and the shortest one $d_{O}(3.66 \pm 0.05 \AA)$ in $\mathrm{CoO}_{6}$ octahedral layers appears in $3 a-\mathrm{LCO}_{2.67}$ due to the lattice constant confinement from $\mathrm{SrTiO}_{3}$ substrates. The highly compressively strained $\boldsymbol{d}$ o makes the structure of $3 a-\mathrm{LCO}_{2.67}$ unstable for accepting more $V_{\ddot{o}}$ and tends to switch towards the $3 c-\mathrm{LCO}_{2.67}$ with a relaxed $d^{\prime} O(3.76 \pm 0.07 \AA)$ and $d^{\prime}{ }_{V o}$ $(4.33 \pm 0.05 \AA)$ along $c$ axis. In the $3 c-\mathrm{LCO}_{2.67}$ on LAO substrate case, the $d_{V o}(3.75 \pm 0.10 \AA)$ and $d_{O}(4.47 \pm 0.12 \AA)$ evolute to be $3.80 \pm 0.11 \AA$ and $4.57 \pm 0.13 \AA$, respectively. We summarize the calculated atomic distances in Table S1. Finally, we define the $d_{V o} / d_{O}$ ratio as $V_{\ddot{o}}$ tolerance factor, which determines the OVC configuration in an epitaxial thin film. The $d_{V o}$ $/ d_{o}$ of $\sim 1.2$ is obtained in the $3 a-\mathrm{LaCoO}_{2.67}$ and $3 c-\mathrm{LaCoO}_{2.67}$ before the orientation switching of OVC. We believe the calculation of this value will serve as a criterion to evaluate the 
orientation stability of OVC in the oxygen-deficient perovskite oxide thin films with similar crystal structure.

In summary, we report the strain-mediated distinct oxygen-ion migration pathways in ultrathin cobaltite thin films using in-situ atomic-resolved STEM imaging. The evolution of OVCs is directly visualized as a function of annealing temperature and annealing time. We reveal the dynamic process of OVCs propagation and orientation switching in $\mathrm{LCO}_{\mathrm{x}}$ films under both tensile and compressive strain. Given a broad interest in the oxygen-ion conductors, we construct a phase diagram of OVC mediated by strain and oxygen content and provide an OVC stability criteria with a conception of tolerance factor. These findings suggest that the dedicated balance between chemical expansion and epitaxial strain dominate the formation and orientation of $\mathrm{OVC}$, providing a practical guide for engineering targeted OVC configuration in oxygen-deficient functional oxide films. Given the abundance of perovskite oxides with ordered OVC, our work offers an exciting opportunity to design pre-determined OVC, especially the vortex-like topological ionic conductive channels, which manifest themselves to engineer unique phases and functionalities in energy materials.

\section{AUTHOR INFORMATION}

\section{Corresponding Author}

*Er-Jia Guo-ejguo@iphy.ac.cn

*Lin Gu-1.gu@iphy.ac.cn

\section{Author Contributions}

$\uparrow$ These authors contribute equally to this work. These samples were grown and processed by Q.J., S.L., and S.R.C. under the guidance of E.J.G.; TEM lamellas were fabricated with FIB milling by F.Q.M; TEM experiments were performed by Q.H.Z., F.Q.M and X.Y.L, analyzed by Q.H.Z., T.T.S and D.S; The first-principles calculations were performed by A.G., E.J.G. 
and L.G. initiated and supervised the work. Q.H.Z. and E.J.G. wrote the manuscript with valuable inputs from L.G. and D.S. All authors participated in revising the manuscript.

\section{ACKNOWLEDGMENT}

This work was supported by the National Key Basic Research Program of China (Grant No. 2020YFA0309100 and No. 2019YFA0308500), the Beijing Natural Science Foundation (Z190010 and 2202060), the Strategic Priority Research Program of Chinese Academy of Sciences (Grant Nos. XDB07030200 and XDB33030200), Key research projects of Frontier Science of Chinese Academy of Sciences (QYZDB-SSW-JSC035), the National Natural Science Foundation of China (Grant Nos. 51672307, 51991344, 11974390, 52025025, 52072400) and the Beijing Nova Program of Science and Technology (Grant No. Z191100001119112).

\section{REFERENCES}

[1] Chroneos, A., Yildiz, B., Tarancon, A., Parfitt, D. \& Kilner, J.A. Oxygen diffusion in solid oxide fuel cell cathode and electrolyte materials: mechanistic insights from atomistic simulations, Energy Environ. Sci. 2011, 4, 2774.

[2] S. Sengodan, S. Choi, A. Jun, T. H. Shin, Y.-W. Ju, H. Y. Jeong, J. Shin, J. T. S. Irvine, and G. Kim, Layered oxygen-deficient double perovskite as an efficient and stable anode for direct hydrocarbon solid oxide fuel cells, Nat. Mater. 2015, 14, 205.

(3) Bishop, S.R. et al. Annual Review of Materials Research, 2014, 44 (ed. D.R. Clarke) 205-239.

[4] Jeen,H., Bi, Z., Choi, W. S., Chisholm, M. F., Bridges, C. A., Paranthaman, M. P., and Lee, H. N. Orienting oxygen vacancies for fast catalytic reaction, Adv. Mater. 2013, 25,6459 .

[5] Waser, R., and Aono, M., Nanoionics-based resistive switching memories, Nat. Mater. 2007, 6, 833 .

[6] Jeen, H., Choi, W. S., Biegalski, M. D., Folkman, C. M., Tung, I. C., Fong, D. D., Freeland, J. W., Shin, D., Ohta, H., Chisholm, M. F., and Lee, H. N. Reversible redox reactions in an epitaxially stabilized $\mathrm{SrCoO}_{x}$ oxygen sponge, Nat. Mater. 2013, 12, 1057. 
[7] Anderson, M.T., Vaughey, J.T. \& Poeppelmeier, K.R. Structural similarities among oxygen-deficient perovskites, Chem. Mater. 1993, 5, 151.

[8] Lu, Q., Chen, Y., Bluhm, H. \& Yildiz, B. Electronic Structure Evolution of SrCoOx during Electrochemically Driven Phase Transition Probed by in Situ X-ray Spectroscopy, J. Phys. Chem. C 2016, 120, 24148.

[9] Cui, B., Werner, P., Ma, T. P., Zhong, X. Y., Wang, Z. C., Taylor, J. M., Zhuang, Y. C., and Parkin, S. S. P., Direct imaging of structural changes induced by ionic liquid gating leading to engineered three-dimensional meso-structures, Nat. Commun. 2018, 9,8 .

[10] Ning,S., Zhang,Q., Occhialini,C., Comin, R., Zhong, X., and Ross, C. A., Voltage Control of Magnetism above Room Temperature in Epitaxial $\mathrm{SrCo}_{1-\mathrm{x}} \mathrm{Fe}_{\mathrm{x}} \mathrm{O}_{3 \text {-delta, }}$ ACS Nano 2020, 14, 8949.

[11] Lu, N., Zhang, P., Zhang, Q., Qiao, R., He, Q., Li, H. B., Wang, Y., Guo, J., Zhang, D., Duan, Z., Li, Z., Wang,M., Yang,S., Yan, M., Arenholz,E., Zhou, S., Yang, W., Gu, L., Nan, C. W., Wu, J., Tokura, Y., and Yu, P., Electric-field control of tri-state phase transformation with a selective dual-ion switch, Nature 2017, 546, 124.

[12] Eckstein, J. N., Oxide interfaces: Watch out for the lack of oxygen, Nat. Mater. 2007, $6,473$.

[13] Gunkel, F., Christensen, D. V., Chen, Y. Z. \& Pryds, N. Oxygen vacancies: The (in)visible friend of oxide electronics, Applied Physics Letters 2020, 116, 7.

[14] Kalinin, S.V. \& Spaldin, N.A. Functional Ion Defects in Transition Metal Oxides, Science 2013, 341, 858.

[15] Xie, Y., Scafetta, M. D., Sichel-Tissot, R. J., Moon, E. J., Devlin, R. C., Wu, H., Krick, A. L., and May, S. J., Control of Functional Responses Via Reversible Oxygen Loss in $\mathrm{La}_{1-\mathrm{x}} \mathrm{Sr}_{\mathrm{x}} \mathrm{FeO}_{3 \text {-delta }}$ Films, Adv. Mater. 2014, 26, 1434.

[16] Inoue,S., Kawai, M. N., Ichikawa, Kageyama, H., Paulus,W. ,and Shimakawa,Y., Anisotropic oxygen diffusion at low temperature in perovskite-structure iron oxides, Nat. Chem. 2010, 2, 213.

[17] Mitra, C., Meyer, T., Lee, H.N. \& Reboredo, F.A. Oxygen diffusion pathways in brownmillerite $\mathrm{SrCoO}_{2.5}$ : Influence of structure and chemical potential, J. Chem. Phys. 2014, 141, 5 .

[18] Islam, M.S., Nolan, A.M., Wang, S., Bai, Q. \& Mo, Y.F. A Computational Study of Fast Proton Diffusion in Brownmillerite Sr2Co2O5, Chem. Mater. 2020, 32, 5028.

[19] Jeen, H., Choi,W. S., Freeland,J. W., Ohta, H., Jung, C. U., and Lee, H. N., 
Topotactic Phase Transformation of the Brownmillerite $\mathrm{SrCoO}_{2.5}$ to the Perovskite SrCoO3-delta, Adv. Mater. 2013, 25, 3651.

[20] Zhang, B. B., He, X., Zhao, J. L., Yu, C., Wen, H. D., Meng, S., Bousquet, E., Li, Y. L. , Ge, C., Jin, K. J., Tao, Y., and Guo, H. Z., Giant photoinduced lattice distortion in oxygen vacancy ordered $\mathrm{SrCoO}_{2.5}$ thin films, Phys. Rev. B 2019, 100, 144201.

[21] An, Q., Meng, M., Wang, Z., Wang, Y., Zhang, Q., Xia, Y., Gu, L., Yang, F., and Guo, J., Realization of Monophased $\mathrm{LaCoO}_{\mathrm{x}}$ Films with Ordered Oxygen Vacancies, Phys. Status Solidi A 2019, 4, 1900848.

[22] Walter, J., Bose, S., Cabero, M., Varela, M. \& Leighton, C. Giant anisotropic magnetoresistance in oxygen-vacancy-ordered epitaxial $\mathrm{La} 0.5 \mathrm{Sr} 0.5 \mathrm{CoO} 3$-delta films, Phys. Rev. Mater. 2020, 4, 091401.

[23] Yang, Q., Cho, H.J., Jeen, H. \& Ohta, H. Macroscopic Visualization of Fast Electrochemical Reaction of SrCoOx Oxygen Sponge, Adv. Mater. Interfaces 2019, 6, 1901260 .

[24] Biskup, N., Salafranca, J., Mehta, V., Oxley, M. P., Suzuki, Y., Pennycook, S. J., Pantelides, S. T., and Varela, M., Insulating Ferromagnetic LaCoO3-delta Films: A Phase Induced by Ordering of Oxygen Vacancies, Phys. Rev. Lett. 2014, 112, 087202.

[25] Saleem, M. S., Song, C., Gu, Y. D., Chen, R. Y., Fayaz, M. U., Hao, Y. M., and Pan, F., Orientation control of oxygen vacancy channels in brownmillerite $\mathrm{SrFeO}_{2.5}$, Phys. Rev. Mater. 2020, 4, 014403.

[26] Khare, A., Lee, J., Park, J., Kim, G. Y., Choi, S. Y., Katase, T., Roh, S., Yoo, T. S., Hwang, J., Ohta, H., Son, J., and Choi, W. S., Directing Oxygen Vacancy Channels in $\mathrm{SrFeO}_{2.5}$ Epitaxial Thin Films, ACS Appl. Mater. Interfaces 2018, 10, 4831.

[27] Nallagatla, V. R., Kim, J., Lee, K., Chae, S. C., Hwang, C. S., and Jung, C. U., Complementary Resistive Switching and Synaptic-Like Memory Behavior in an Epitaxial $\mathrm{SrFeO}_{2.5}$ Thin Film through Oriented Oxygen-Vacancy Channels, ACS Appl. Mater. Interfaces 2020, 12, 41740.

[28] Petrie, J. R., Mitra, C., Jeen, H., Choi, W. S., Meyer, T. L., Reboredo, F. A., Freeland, J. W., Eres, G., and Lee, H. N., Strain Control of Oxygen Vacancies in Epitaxial Strontium Cobaltite Films, Adv. Func. Mater. 2016, 26, 1564.

[29] Zhang, K. H. L., Sushko, P. V., Colby, R., Du, Y., Bowden, M. E., and Chambers, A., Reversible nano-structuring of $\mathrm{SrCrO} 3$-delta through oxidation and reduction at low temperature, Nat. Commun. 2014, 5, 7.

[30] Wang, L., Yang, Z., Bowden, M. E., and Du, Y., Brownmillerite phase formation and 
evolution in epitaxial strontium ferrite heterostructures, Appl. Phys.s Lett. 2019, 114, 231602.

[31] Yamamoto, T., Chikamatsu, A., Kitagawa, S., Izumo, N., Yamashita, S., Takatsu, H., Ochi, M., Maruyama, T., Namba, M., Sun, W., Nakashima, T., Takeiri, F., Fujii, K., Yashima, M., Sugisawa, Y., Sano, M., Hirose, Y., Sekiba, D., Brown, C. M., Honda, T., Ikeda, K., Otomo, T., Kuroki, K., Ishida, K., Mori, T., Kimoto, K., Hasegawa, T., and Kageyama, H., Strain-induced creation and switching of anion vacancy layers in perovskite oxynitrides, Nat. Commun. 2020, 11, 5923.

[32] Zhang, Q., Gao, A., Meng, F., Jin, Q., Lin, S., Wang, X., Xiao, D., Wang, C., Jin, K. J., Su, D., Guo, E. J., and Gu, L., Near-room temperature ferromagnetic insulating state in highly distorted $\mathrm{LaCoO}_{2.5}$ with $\mathrm{CoO}_{5}$ square pyramids, Nat. Commun. 2021, 12 , 1853.

[33] Jang, J. H., Kim, Y. M., He, Q., Mishra, R., Qiao, L., Biegalski, M. D., Lupini, A. R., Pantelides, S. T., Pennycook, S. J., Kalinin, S. V., and Borisevich, A. Y., In Situ Observation of Oxygen Vacancy Dynamics and Ordering in the Epitaxial $\mathrm{LaCoO}_{3}$ System, ACS Nano 2017, 11, 6942.

[34] Lan, Q. Q., Zhang, X. J., Shen, X., Yang, H. W., Zhang, H. R., Guan, X. X., Wang, W., Yao, Y., Wang, Y. G., Peng, Y., Liu, B. G., Sun, J. R., and Yu, R. C., Tuning the magnetism of epitaxial cobalt oxide thin films by electron beam irradiation, Phys. Rev. Mater. 2017, 1, 024403.

[35] Kim, Y.-M., Morozovska, A., Eliseev, E., Oxley, M. P., Mishra, R., Selbach, S. M., Grande, T., Pantelides, S. T., Kalinin, S. V., and Borisevich, A. Y., Direct observation of ferroelectric field effect and vacancy-controlled screening at the $\mathrm{BiFeO}_{3} / \mathrm{La}_{x} \mathrm{Sr}_{1}$ ${ }_{x} \mathrm{MnO}_{3}$ interface, Nat. Mater. 2014, 13, 1019.

[36] Herklotz, A., Lee, D., Guo, E. J., Meyer, T. L., Petrie, J. R., and Lee, H. N., Strain coupling of oxygen non-stoichiometry in perovskite thin films, J. Phys. -Conden. Matter. 2017, 29, 493001.

[37] Aschauer, U., Pfenninger, R., Selbach, S.M., Grande, T. \& Spaldin, N.A. Straincontrolled oxygen vacancy formation and ordering in $\mathrm{CaMnO}_{3}$, Phys. Rev. B 2013, 88, 054111 .

[38] Marthinsen, A., Faber, C., Aschauer, U., Spaldin, N.A. \& Selbach, S.M. Coupling and competition between ferroelectricity, magnetism, strain, and oxygen vacancies in $\mathrm{AMnO}_{3}$ perovskites, MRS Commun. 2016, 6, 182.

[39] Chandrasena, R. U., Yang, W., Lei, Q., Delgado-Jaime, M. U., Wijesekara, K. D., 
Golalikhani, M., Davidson, B. A., Arenholz, E., Kobayashi, K., Kobata, M., de Groot, F. M. F., Aschauer, U., Spaldin, N. A., Xi, X., and Gray, A. X., Strain-Engineered Oxygen Vacancies in $\mathrm{CaMnO}_{3}$ Thin Films, Nano Letters 2017, 17, 794.

[40] Agrawal, P., Guo, J., Yu, P., Hébert, C., Passerone, D., Erni, R., and Rossell, M. D., Strain-driven oxygen deficiency in multiferroic $\mathrm{SrMnO}_{3}$ thin films, Phys. Rev. B 2016, 94, 104101.

[41] Choi, W. S., Kwon, J.-H., Jeen, H., Hamann-Borrero, J. E., Radi, A., Macke, S., Sutarto, R., He, F., Sawatzky, G. A., Hinkov, V., Kim, M., and Lee, H. N., StrainInduced Spin States in Atomically Ordered Cobaltites, Nano Letters 2012, 12, 4966.

[42] Guo, E.-J., Desautels, R., Lee, D., Roldan, M. A., Liao, Z., Charlton, T., Ambaye, H., Molaison, J., Boehler, R., Keavney, D., Herklotz, A., Ward, T. Z., Lee, H. N., and Fitzsimmons, M. R., Exploiting Symmetry Mismatch to Control Magnetism in a Ferroelastic Heterostructure, Phys. Rev. Lett. 2019, 122, 187202.

[43] Li, S., Wang, J., Zhang, Q., Roldan, M. A., Shan, L., Jin, Q., Chen, S., Wu, Z., Wang, C., He, M., Guo, H., Gu, L., Jin, K.., and Guo, E.-J., Maximization of ferromagnetism in $\mathrm{LaCoO}_{3}$ films by competing symmetry, Phys. Rev. Mater. 2019, 3, 114409.

[44] Zhang, N., Zhu, Y., Li, D., Pan, D., Tang, Y., Han, M., Ma, J., Wu, B., Zhang, Z., and Ma, X., Oxygen Vacancy Ordering Modulation of Magnetic Anisotropy in Strained LaCoO3-x Thin Films, Acs Appl. Mater. Inter. 2018, 10, 38230.

[45] Kwon, J.-H., Choi, W. S., Kwon, Y.-K., Jung, R., Zuo, J.-M., Lee, H. N., and Kim, M., Nanoscale Spin-State Ordering in $\mathrm{LaCoO}_{3}$ Epitaxial Thin Films, Chem. Mater. 2014, 26, 2496.

[46] Guo, E. J., Liu, Y. H., Sohn, C., Desautels, R. D., Herklotz, A., Liao, Z. L., Nichols, J., Freeland, J. W., Fitzsimmons, M. R., and Lee, H. N., Oxygen diode formed in nickelate heterostructures by chemical potential mismatch, Adv. Mater. 2018, 30, 1705904.

[47] Mayeshiba, T. T., and Morgan, D. D., Factors controlling oxygen migration barriers in perovskites, Solid State Ionics 2016, 296, 71.

[48] Lontsi-Fomena,M., Villesuzanne, A., Doumerc, J. P., Frayret, C., and Pouchard, M., A density functional theory study of oxygen diffusion in $\mathrm{LaAlO}_{3}$ and $\mathrm{SrTiO}_{3}$, Comput. Mater. Sci. 2008, 44, 53.

[49] Hansteen, O.H., Fjellvag, H. \& Hauback, B.C. Crystal structure, thermal and magnetic properties of $\mathrm{La}_{3} \mathrm{Co}_{3} \mathrm{O}_{8}$. Phase relations for $\mathrm{LaCoO}_{3 \text {-delta }}(0.00<=$ delta $<=0.50)$ at 673 K, J. Mater. Chem. 1998, 8, 2081. 

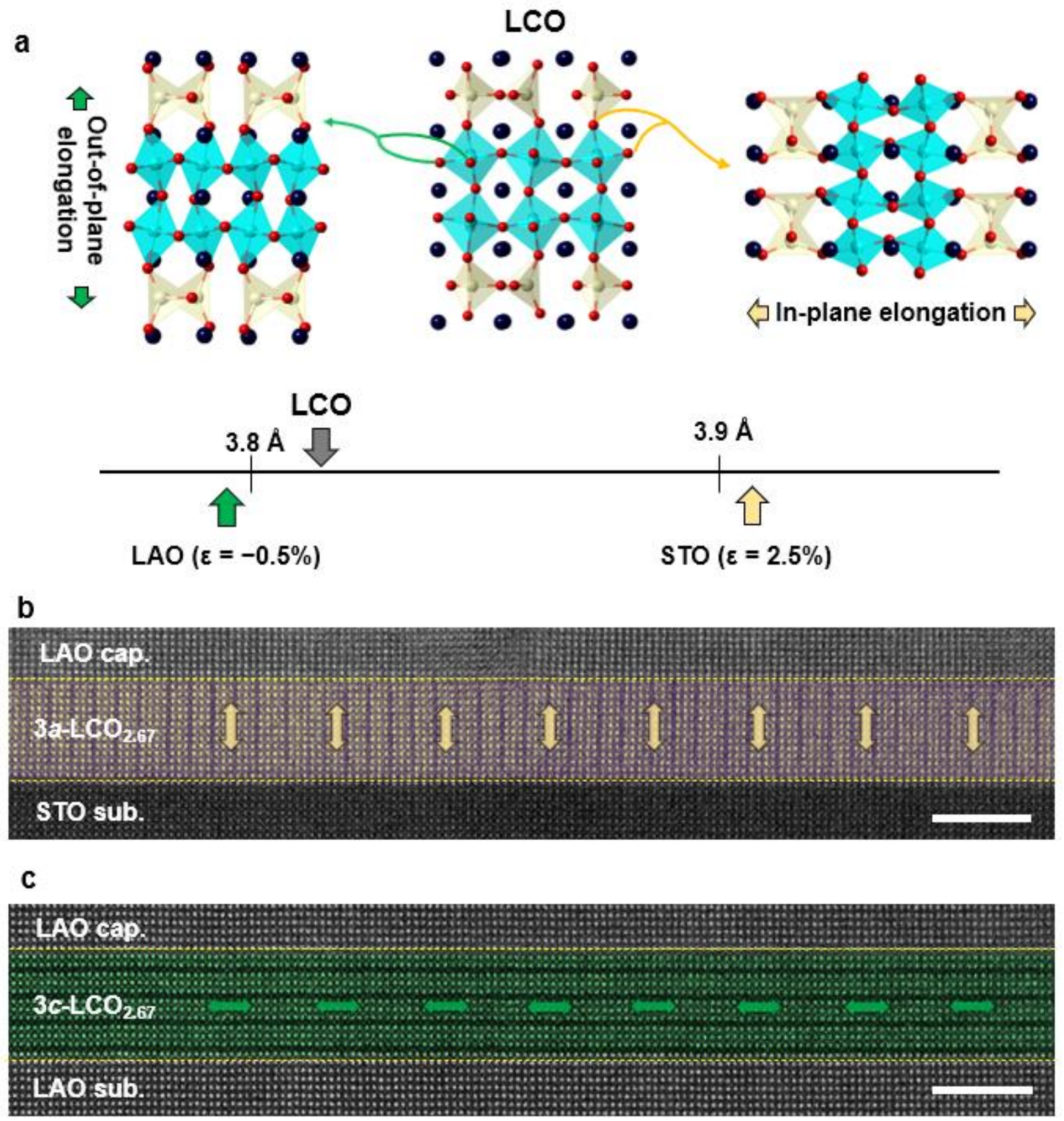

\section{PTIONS}

Figure 1. Direct observations of OVCs on strained LCOx films. (a) Schematics of OVCs evolution on the $\mathrm{LaAlO}_{3}(\mathrm{LAO})$ and $\mathrm{SrTiO}_{3}$ (STO) substrates. The $\mathrm{LCO}_{\mathrm{x}}$ films will form alternated tetrahedron $\left[\mathrm{CoO}_{4}\right.$, yellow] and octahedron $\left[\mathrm{CoO}_{6}\right.$, blue] along the in-plane (out-ofplane) direction under tensile (compressive) strain. (b) and (c) HAADF images of $\mathrm{LCO}_{2.67}$ films on $\mathrm{SrTiO}_{3}$ and $\mathrm{LaAlO}_{3}$ substrates, respectively. $3 a-\mathrm{LCO}_{2.67}\left(3 c-\mathrm{LCO}_{2.67}\right)$ represent the domain orientation aligned out-of-plane (in-plane) direction. 

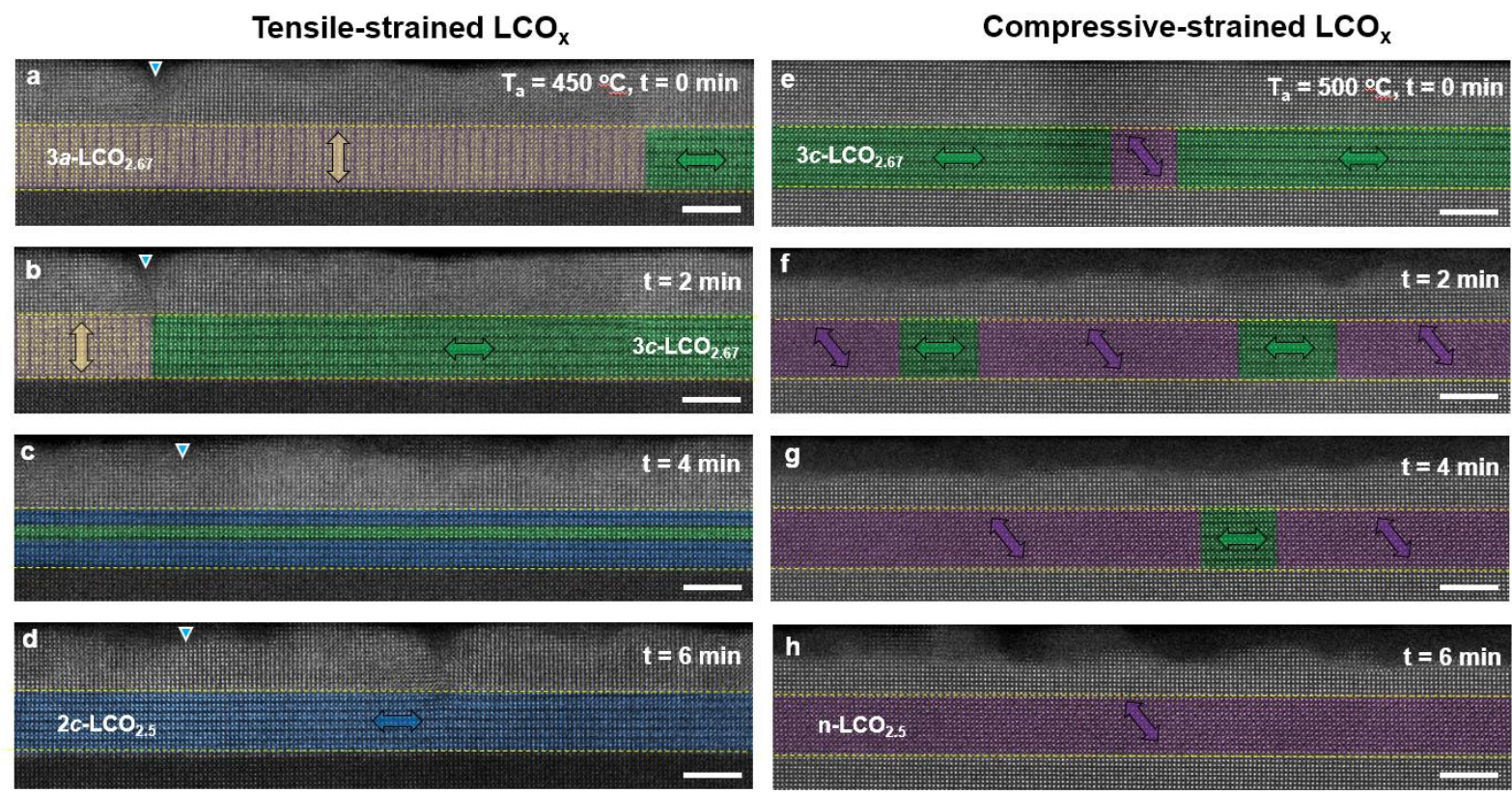

Figure 2. Structural evolution of OVCs in strained LCO $_{\mathbf{x}}$ ultrathin films. Both specimens were in-situ heated up from room-temperature to annealing temperature $T_{\mathrm{a}}$. (a)-(d) HAADF images of a tensile-strained $\mathrm{LCO}_{\mathrm{x}}$ film taken after annealing at $T_{\mathrm{a}}=450{ }^{\circ} \mathrm{C}$ for $0-, 2-, 4-$, and 6-minutes waiting time. The $3 c-\mathrm{LCO}_{2.67}$ and $3 a-\mathrm{LCO}_{2.67}$ domains are marked in green and yellow, respectively. After 4 minutes, the $\mathrm{LCO}_{\mathrm{x}}$ film transforms into the brownmillerite $2 c$ $\mathrm{LCO}_{2.5}$ partially, then changes into $2 c$ - $\mathrm{LCO}_{2.5}$ (blue domains) entirely after 6 minutes. The white triangle indicates the positions of the edge dislocation. (e)-(h) HAADF images of a compressively strained $\mathrm{LCO}_{\mathrm{x}}$ film taken consequently after annealing at $T_{\mathrm{a}}=500{ }^{\circ} \mathrm{C}$ for $0-, 2-$, 4-, and 6-minutes waiting time. The $3 c$ - $\mathrm{LCO}_{2.67}$ transforms into a new $\mathrm{LCO}_{2.5}\left(\mathrm{n}-\mathrm{LCO}_{2.5}\right)$ phase (purple domains). With increasing the annealing time, all $\mathrm{n}-\mathrm{LCO}_{2.5}$ phase merges. The white scale bar is $5 \mathrm{~nm}$. Yellow dashed lines indicate the interface positions. 
a

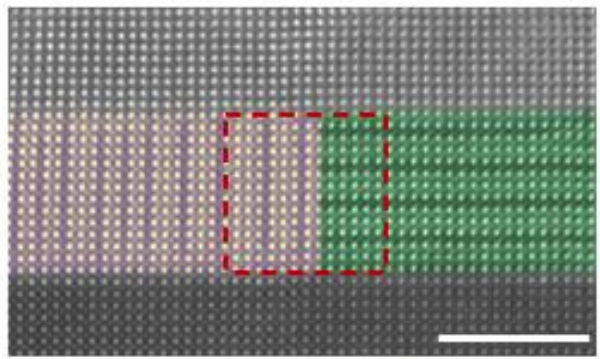

b

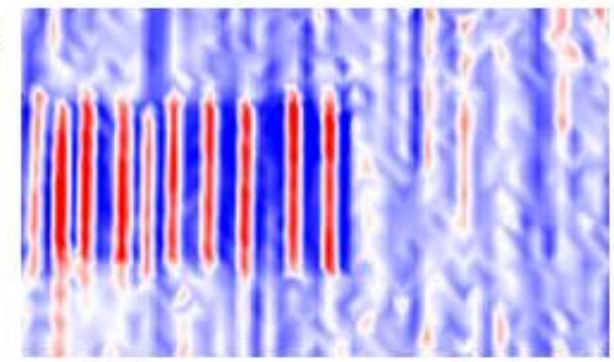

$3.5 \mathrm{~A}$

d

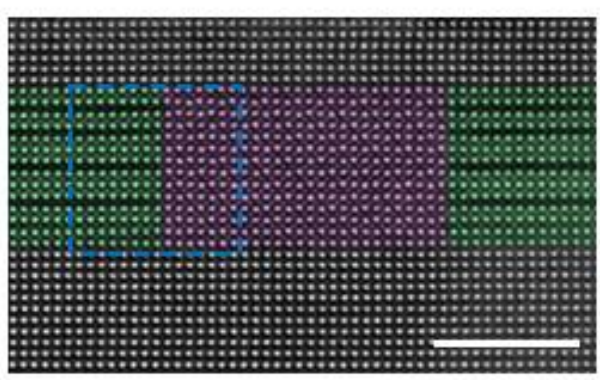

e

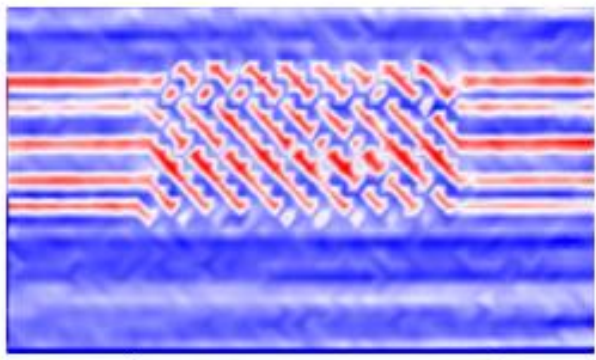

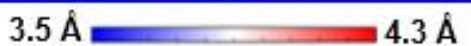

Domain boundary

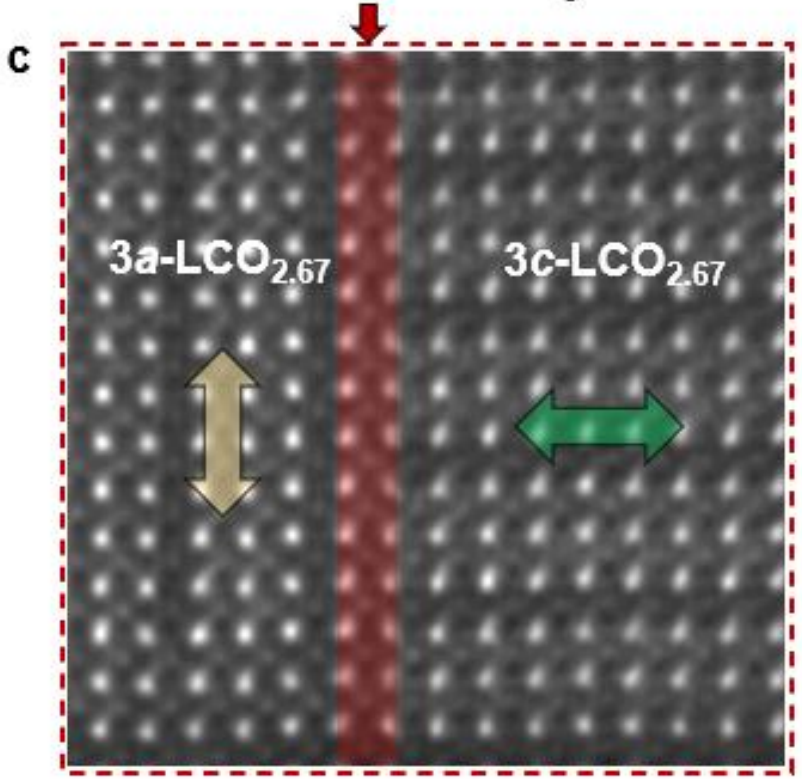

\section{Domain boundary}

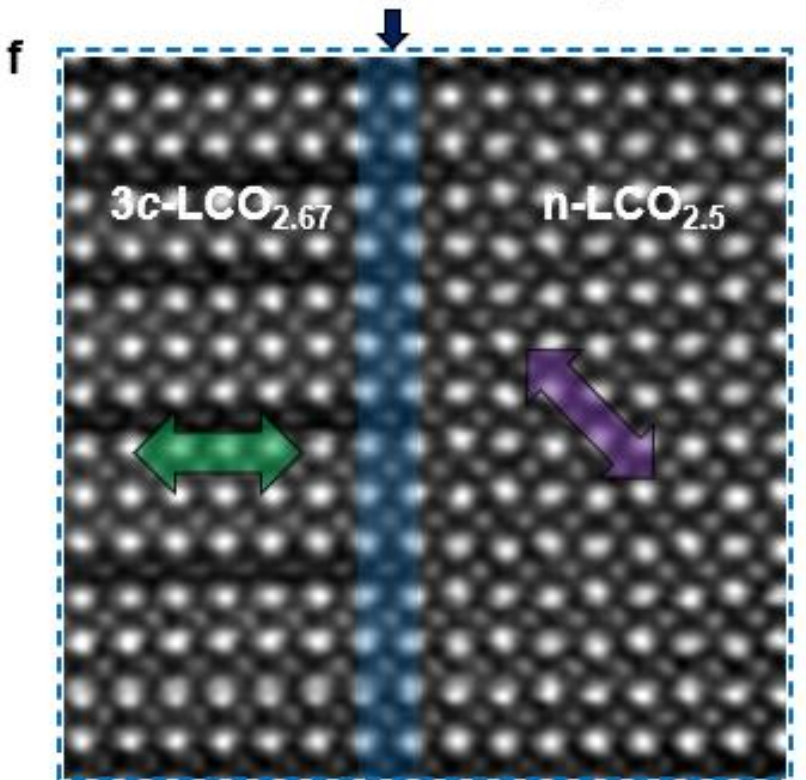

Figure 3. Microscopic view and strain distribution of domain boundaries in $\mathrm{LCO}_{\mathrm{x}}$ ultrathin films. HAADF images of domain boundaries (a) between $3 a-\mathrm{LCO}_{2.67}$ and $3 c$ - $\mathrm{LCO}_{2.67}$ in a tensile-strained film and (d) $3 c-\mathrm{LCO}_{2.67}$ and n- $\mathrm{LCO}_{2.5}$ in a compressive strained film. Highmagnified HAADF images from red and blue squares are shown in (c) and (f), where boundaries of OVC switching are indicated by red and blue colors, respectively. The in-plane and out-ofplane lattice strain mapping corresponding to (a) and (d) are shown in (b) and (e), respectively. 


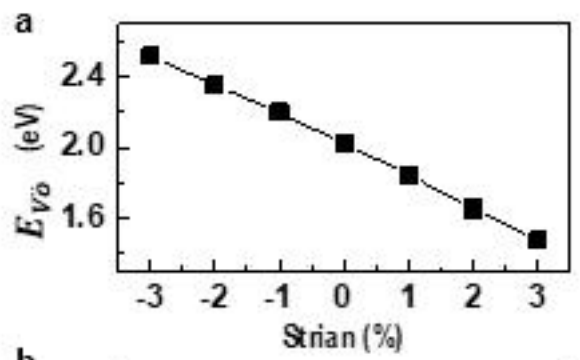

C
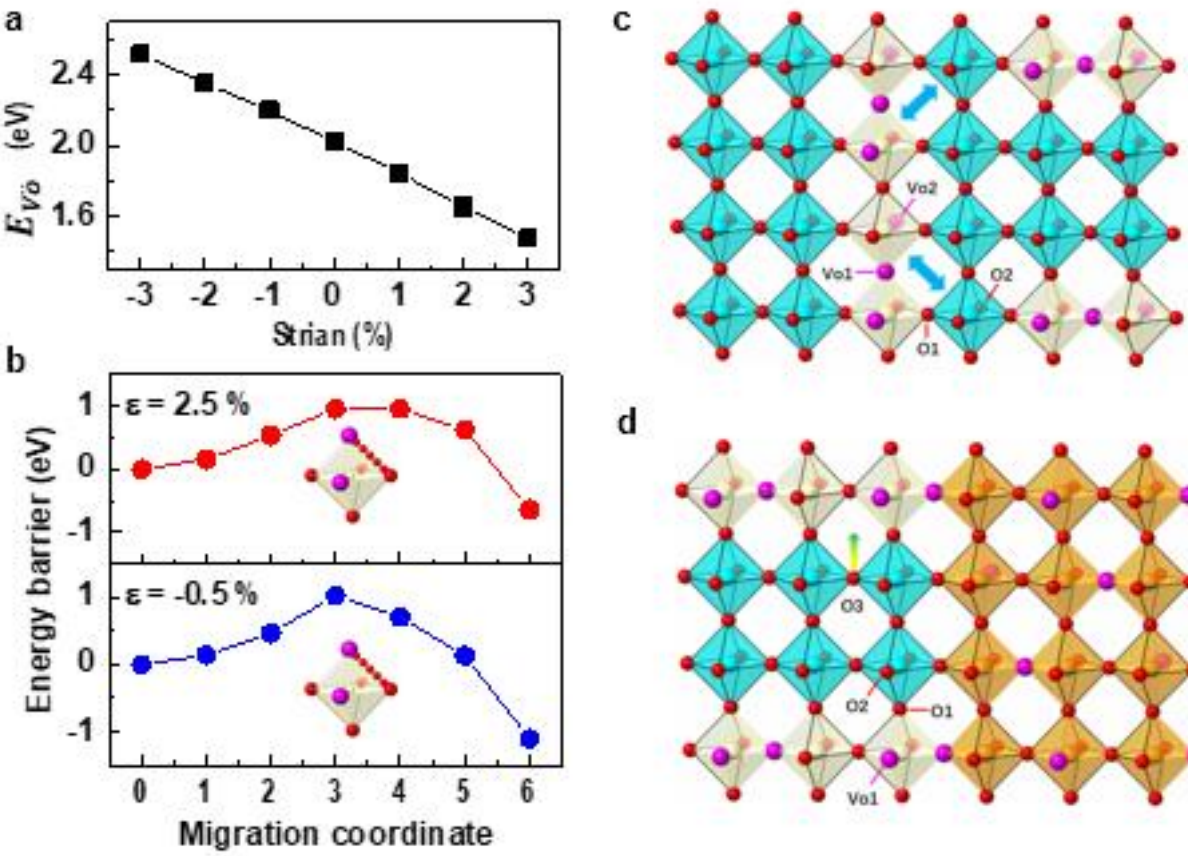

d
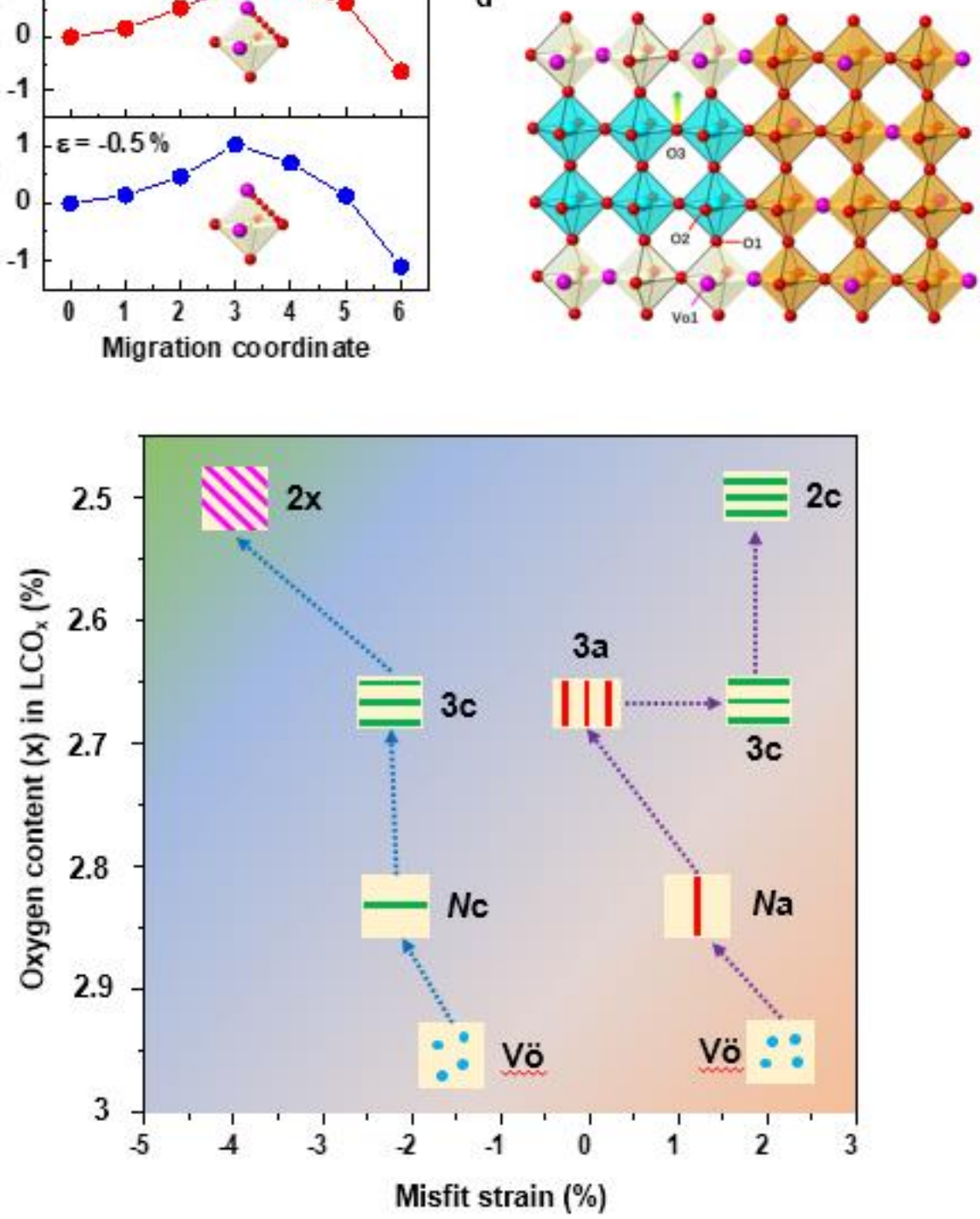

Figure 4. Strain dependent oxygen migration and phase diagram of OVCs in cobaltites. (a) Oxygen vacancy formation energy $\left(E_{V o ̈}\right)$ in $\mathrm{LaCoO}_{3}$ film mediated by substrate strain. (b) The migration barrier in the $\mathrm{CoO}_{4}$ tetrahedra under tensile (upper panel) and compressive strain (lower panel). Oxygen-ion migration pathway when a $\mathrm{LCO}_{\mathrm{x}}$ film under (c) tensile and (d) compressive strain. Red and purple spheres represent oxygen-ions and oxygen vacancies, respectively. (e) Phase diagram of OVCs. The oxygen content (x) is plotted as a function of misfit strain, which is caused by $V_{\ddot{o}}$-induced chemical expansion. $N \mathrm{a}$ and $N \mathrm{c}$ are two intermedia states of $\mathrm{LCO}_{\mathrm{x}}$ films during the phase transition under tensile and compressive strains, respectively. Note that the misfit strain and corresponding oxygen content are calculated directly from the STEM measurements. 


\section{TOC graphics}

Tensile strain

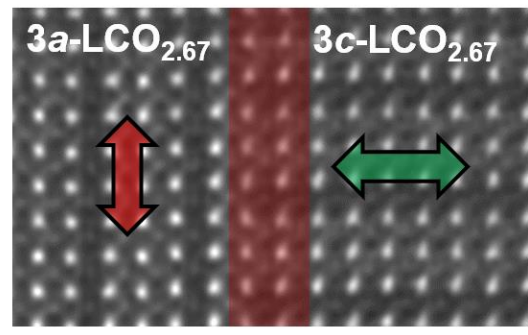

Compressive strain

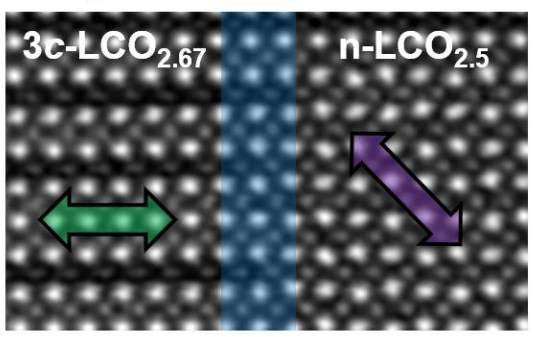

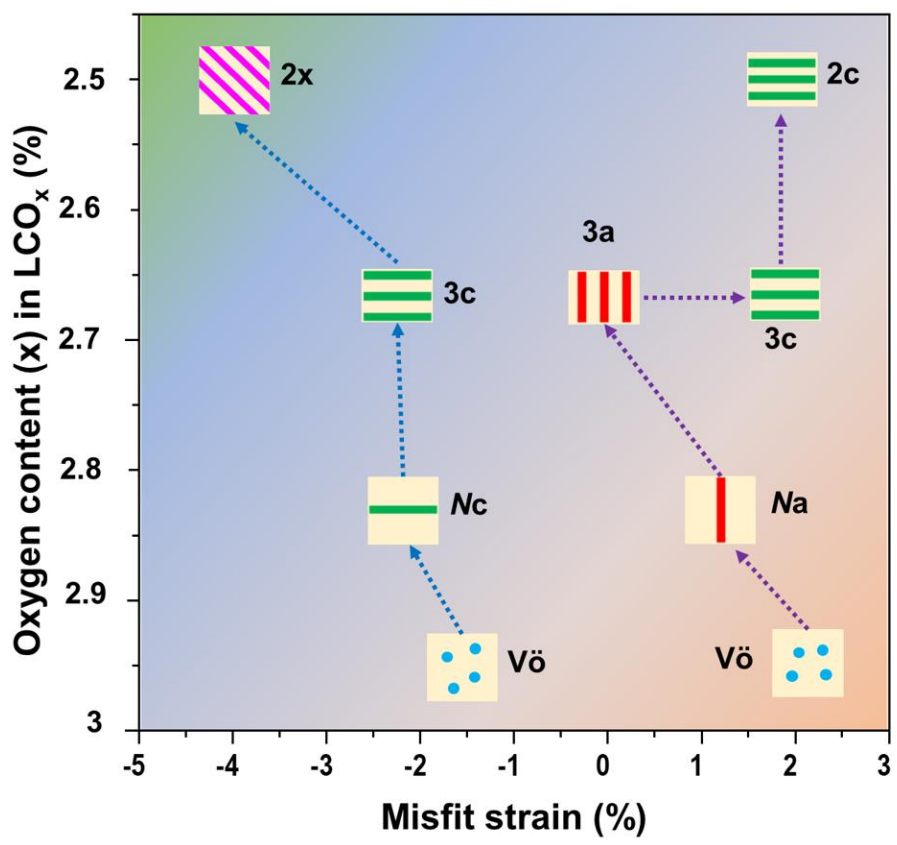

Dynamic anisotropic oxygen-ion migration in cobaltite thin films is observed at atomicscale via STEM. A phase diagram of orientation of oxygen vacancy (OVC) mediated by strain and oxygen content, and an OVC stability criteria are constructed, providing a practical guide for engineering targeted OVC configuration in oxygen-deficient functional oxide films. 